\title{
The Vibrational Spectra of Succinimide and $\boldsymbol{N}$-Deuteriosuccinimide
}

\author{
T. WOLDBAE, ${ }^{a}$ P. KLAEBOE ${ }^{a}$ and D. H. CHRISTENSEN ${ }^{b}$
} a Department of Chemistry, University of Oslo, Oslo 3, Norway and b Chemical Laboratory V, The H. C.
Ørsted Institute, DK-2100, Copenhagen, Denmark

The infrared spectra $\left(4000-200 \mathrm{~cm}^{-1}\right)$ of succinimide as a vapour $\left(160^{\circ} \mathrm{C}\right)$, melt $(\sim 130$ $\left.{ }^{\circ} \mathrm{C}\right)$, oriented polycrystalline film, KT-pellet, $\mathrm{Nujol}$ mull and dissolved in $\mathrm{CCl}_{4}, \mathrm{CS}_{2}, \mathrm{CH}_{2} \mathrm{Cl}_{2}$, and $\mathrm{CH}_{3} \mathrm{CN}$ were recorded. Additional spectra were obtained in the region $400-30 \mathrm{~cm}^{-1}$. Raman spectra of the crystalline solid and saturated solutions in various polar solvents were recorded and semiquantitative polarization ratios were measured. Infrared and Raman spectra of $N$-deuteriosuccinimide in the solid state were recorded.

The fundamental frequencies were tentatively assigned in terms of $C_{2 v}$ symmetry based upon Raman polarization data, dichroism of the oriented film and analogies with the spectra of the $N$-halogenated succinimides. Force fields were derived by initially transferring force constants from maleimide and succinic anhydride, and the data were fitted together with those of the N-halogenated succinimides by a least square method.

We have recently reported the vibrational spectra of maleic anhydride, ${ }^{1}$ maleimide, ${ }^{2}$ and $N$-chloromaleimide. ${ }^{3}$ These studies have now been extended to the corresponding saturated cyclic imides. In the present communication we shall report our results for succinimide (SIM) and the $N$-deuterated species (SIMD), while the $N$-halogenated derivatives will be treated in a forthcoming paper. ${ }^{3}$ Previously, infrared studies of SIM and SIMD as solids have been restricted to the $\mathrm{KBr}$ region ${ }^{5}$ and oriented crystals in the $\mathrm{NaCl}$ region. ${ }^{6}$ No melt or solution data have been reported and the Raman spectra have to our knowledge not been recorded.

In the present study we have obtained as complete infrared and Raman data as possible within the limitations imposed by the low vapour pressure and restricted solubility of
SIM. The assigned fundamentals were compared with the results of a normal coordinate analysis, using a generalized valence force field, and treating SIM and SIMD together with the $N$ halogenated succinimides. ${ }^{4}$

\section{EXPERIMENTAL}

The sample of SIM was a commercial product from Fluka $A G$, which was purified by repeated sublimation on a cold finger (m.p. $126^{\circ} \mathrm{C}$, b.p. $287^{\circ} \mathrm{C}$ ). The deuterated compound (SIMD) was prepared by successive treatments with $\mathrm{D}_{2} \mathrm{O}$ and evaporation of the water. Although most of the sample handling took place in a dry box, a slight exchange of deuterium from moist air was observed in the infrared spectra.

The infrared, far infrared, and Raman (using the 4880 and $5145 \AA$ lines from an argon ion laser (CRL $52 \mathrm{G}$ )) spectrometers, ${ }^{1}$ cells, and general procedures ${ }^{2}$ have been described.

Dimethylformamide (DMFA) was used as solvent for the Raman spectra because of the low solubility of SIM in non-polar solvents.

\section{RESULTS}

SIM and certain $N$-halogenated succinimides have been studied by $X$-ray crystallography and the ring skeleton reported ${ }^{7,8}$ to be nearly planar. Furthermore, the same holds true for the related succinic anhydride in the vapour phase.

The fact that SIM forms dimeric molecules in the crystal and probably in solution because of two $\mathrm{N}-\mathrm{H} . \mathrm{O} \mathrm{O}=\mathrm{C}$ hydrogen bonds (contrary to the $N$-halogenated derivatives) presumably do not perturb the fundamentals significantly. As previously noted for the maleimides 2,8 the comparatively weak intermolecular bonds result in coinciding $a_{g}$ and $b_{u}$ as well as $a_{w}$ 
and $b_{g}$ modes ( $C_{2 h}$ symmetry of the dimer). Only the $\mathrm{N}-\mathrm{H}$ stretching and bending modes are highly affected by the hydrogen bonding. Therefore it seems appropriate to assume that SIM and SIMD (like the corresponding maleimide ${ }^{2}$ and maleic anhydride ${ }^{1}$ ) have $C_{2 v}$ sym. metry in all the states of aggregation. The 30 fundamentals will then divide themselves into the symmetry species: $10 a_{1}+5 a_{2}+6 b_{1}+9 b_{2}$ in which $a_{1}$ and $b_{2}$ represent in-plane, $a_{2}$ and $b_{1}$ out-of-plane modes. All the fundamentals are active in the Raman effect while those of species $a_{2}$ are forbidden in the infrared spectra.

A vapour IR spectrum of SIM was recorded at $c a .160^{\circ} \mathrm{C}$, but due to the low vapour pressure, the vapour contours gave no relevant information for the assignments. Instead, infrared measurements of the dichroic ratio of oriented polycrystalline films gave in many cases direct information about the species. This method, particularly suitable for orthorhombic crystals, has previously been applied to succinimide by Hayashii ${ }^{\circ}$ and will therefore not be described here. Our dichroic measurements of various oriented polycrystalline films of SIM were much more complete in terms of spectral resolution and frequency range than the earlier work ${ }^{\circ}$ employing $\mathrm{NaCl}$-optics, but confirmed the earlier results.

Under the assumption of $C_{2 v}$ symmetry for the molecules, the transition moments will be directed along the $X$-, $Y$ - and $Z$-axes (Fig. 2) for the $b_{1} b_{2}$ and $a_{1}$ modes, respectively. If an oriented gas model can be applied, the dichroic ratio should be equal for all vibrations belonging to the same species. In accordance with Hayashii's ${ }^{6}$ determination of the crystal axes, the following infrared intensity ratios should be expected for SIM:

(a) $I_{b}>I_{c}>I_{a}$ for $a_{1}$

(b) $I_{c}>I_{a}>I_{b}$ for $b_{2}$

(c) $I_{a}>\left(I_{b}\right.$ and $\left.I_{c}\right)$ for $a_{1}$ vibrations.

$I_{a}, I_{b}$ and $I_{c}$ denote the relative intensities for the three mutually perpendicular axes $a, b$ and $c$ of the orthorhombic unit cell.

Additional support for the assignments was provided by the Raman polarization data obtained in saturated solutions in dimethyl. formamide and water. Also, the force constant calculations of SIM and SIMD were of considerable aid. Moreover, the striking similarity between the spectra of SIM and those of $N$. chloro-, $N$-bromo-, and $N$-iodosuccinimide was of great help for the assignments.

Spectral interpretations. As an illustration, the infrared spectra of two oriented poly. crystalline films of SIM are shown in Figs. IA ( $E$ vector parallell with the $c$ and $a$ axes) and IB ( $E$ vector parallell with the $b$ and $a$ axes). The Raman spectrum of the solid is given in Fig. 1C, while the observed infrared and Raman frequencies are listed in Tables 1 (SIM) and 2 (SIMD). Our assigned fundamentals for both compounds are given in Table 3 together with the calculated frequencies.

The broad, intense bands at 3150 (solid), 3270 (melt) and $3280 \mathrm{~cm}^{-1}$ ( $\mathrm{CH}_{3} \mathrm{CN}$ solution) were interpreted as the hydrogen bonded $\mathrm{N}-\mathrm{H}$ stretch $\left(v_{1}\right)$. In $\mathrm{CCl}$, solution an infrared band at $3423 \mathrm{~cm}^{-1}$ was considered as the "free" $\nu_{1}$ (Table 1.) An intense infrared band at 3070 $\mathrm{cm}^{-1}$ (solid) (absent for SIMD) disappeared in dilute solution and was assigned ${ }^{5}$ as $\nu_{23}+$ $v_{24}$ in Fermi resonance with $v_{1}$. In SIMD the hydrogen bonded $\nu_{1}$ was found at $2326 \mathrm{~cm}^{-1}$ in the solid. The present data suggest that the hydrogen bonding is stronger in SIM than in maleimide since the $\mathrm{N}-\mathrm{H}$ frequency shifts from dilute solution to melt and further to the solid state were larger for SIM. The two $\mathrm{CH}_{2}$ groups of SIM and SIMD will give rise to four $\mathrm{C}-\mathrm{H}$ stretching frequencies (one of each species) which were attributed to bands between 3000 and $2940 \mathrm{~cm}^{-1}$ in SIM, SIMD and the $N$-halogenated succinimides."

It is well known from the maleimides, ${ }^{2,3}$ maleic, ${ }^{1,10}$ and succinic anhydride ${ }^{10}$ that the $\mathrm{C}=\mathrm{O}$ stretch of species $a_{1}$ is invariably at higher frequency than the $b_{2}$ mode. The same rule applies to SIM, SIMD and the $N$-halogenated succinimides and will be discussed in the forthcoming paper." In SIM $v_{2}$ and $v_{23}$ were found at 1772 and $1697 \mathrm{~cm}^{-1}$, whereas in SIMD they were situated at 1771 and $1674 \mathrm{~cm}^{-1}$, respectively. A strong Raman band at $\mathbf{1 7 6 6}$ $\mathrm{cm}^{-1}$ in SIMD was interpreted as $v_{8}+v_{10}$ in Fermi resonance with $v_{3}$. The present molecules as well as the corresponding maleimides 2,3 and anhydrides ${ }^{1,10}$ have several strong or medium intense bands in the region assigned

Acta Chem. Scand. A 30 (1976) No. 7 

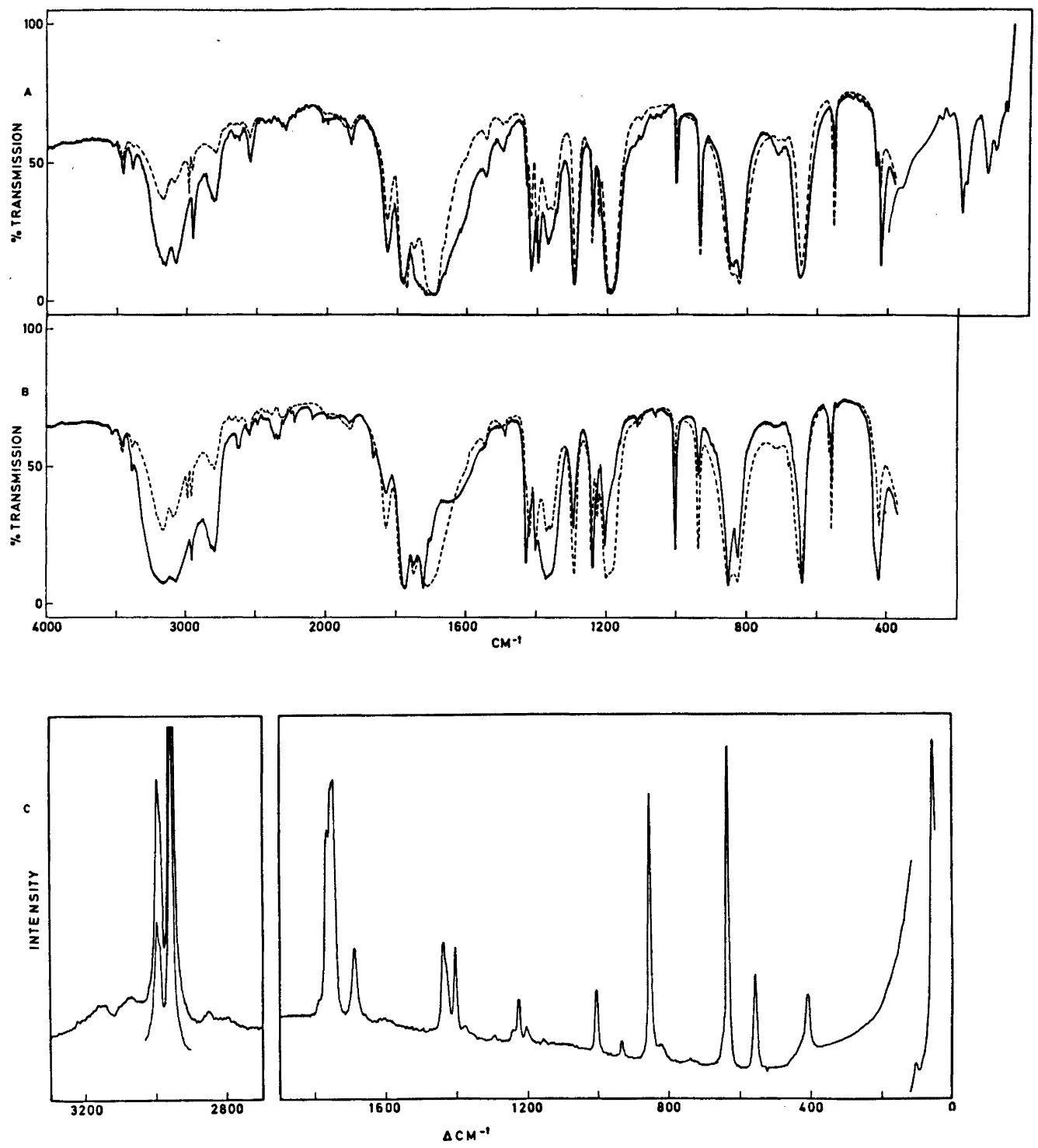

Fig. 1. Infrared and Raman spectra of succinimide in the solid state: A. (upper curve) 4000-400 $\mathrm{cm}^{-1}$ : the infrared spectrum of oriented crystals between $\mathrm{KBr}$ plates; solid line, $E$ vector along $c$ axis; dotted line, $E$ vector along $a$ axis. $400-30 \mathrm{~cm}^{-1}$ : polyethylene pellet; B. (middle curve) the infrared spectrum of oriented crystals between $\mathrm{KBr}$ plates; solid line, $E$ vector along $b$ axis; dotted line, $E$ vector along $a$ axis; C. (lower curve) the Raman spectrum.

to combination bands or overtones, partly enhanced by Fermi resonance to the $a_{1}$ and $b_{2}$ fundamentals.

The two methylene groups of SIM and SIMD will give rise to two scissor $\left(a_{1}, b_{2}\right)$, wag $\left(a_{1}, b_{2}\right)$, twist $\left(a_{2}, b_{1}\right)$ and rock $\left(a_{2}, b_{1}\right)$ fundamentals Acta Chem. Scand. A 30 (1976) No. 7 of which the scissor and wag are generally localized vibrations situated around 1450 and $1300 \mathrm{~cm}^{-1}$, respectively. All the succinimides had intense infrared and Raman bands around $1425 \mathrm{~cm}^{-1}$ assigned to an $a_{1}$ mode. The corresponding $b_{2}$ scissoring modes $\left(v_{25}\right)$ were assigned 


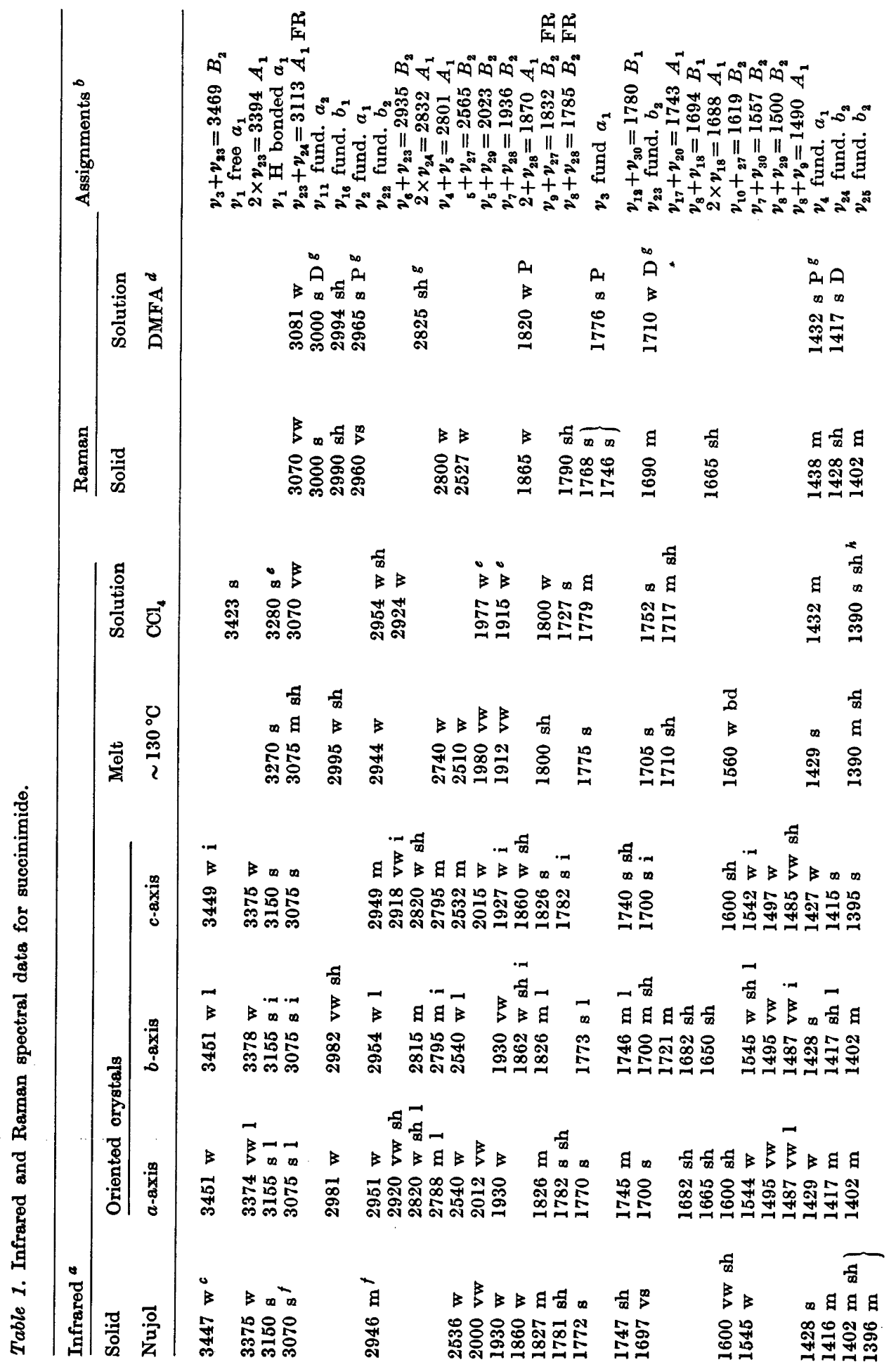




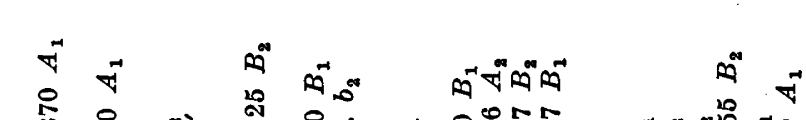
O.

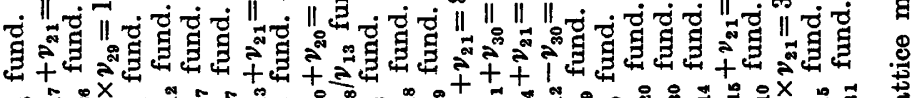

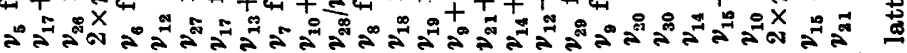

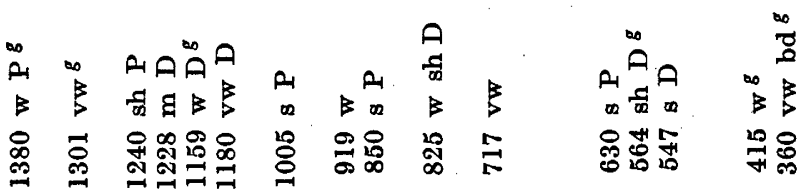

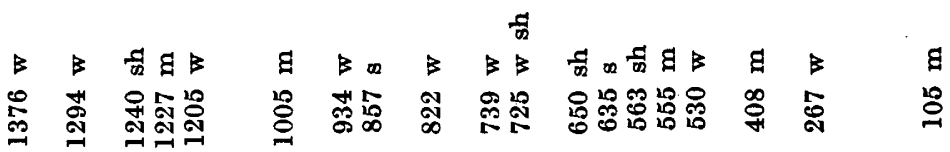

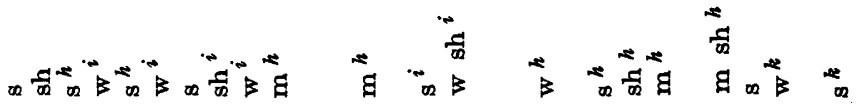

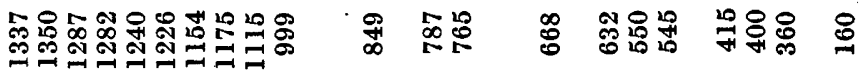

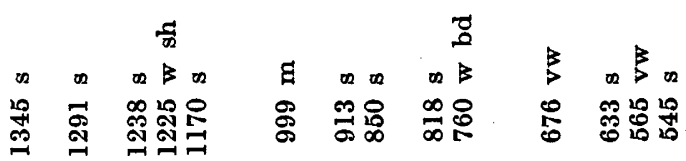

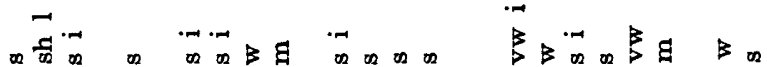

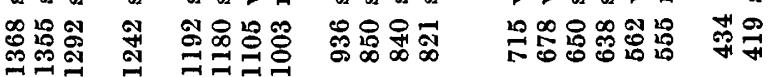

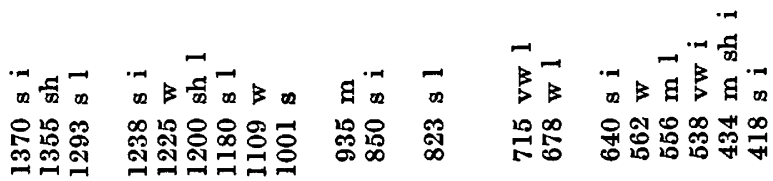

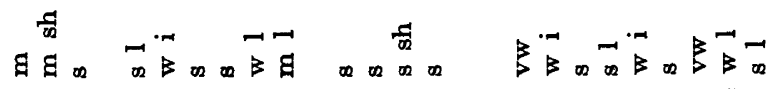

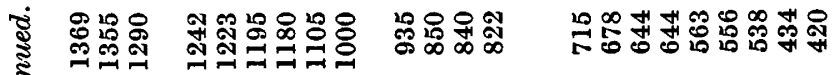

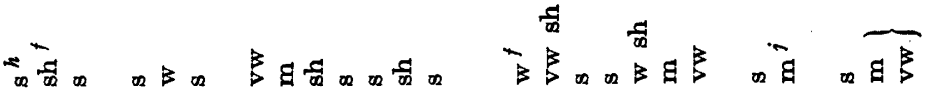
苛 
Table 2. Infrared and Raman spectral data for $N$-deuteriosuccinimide.

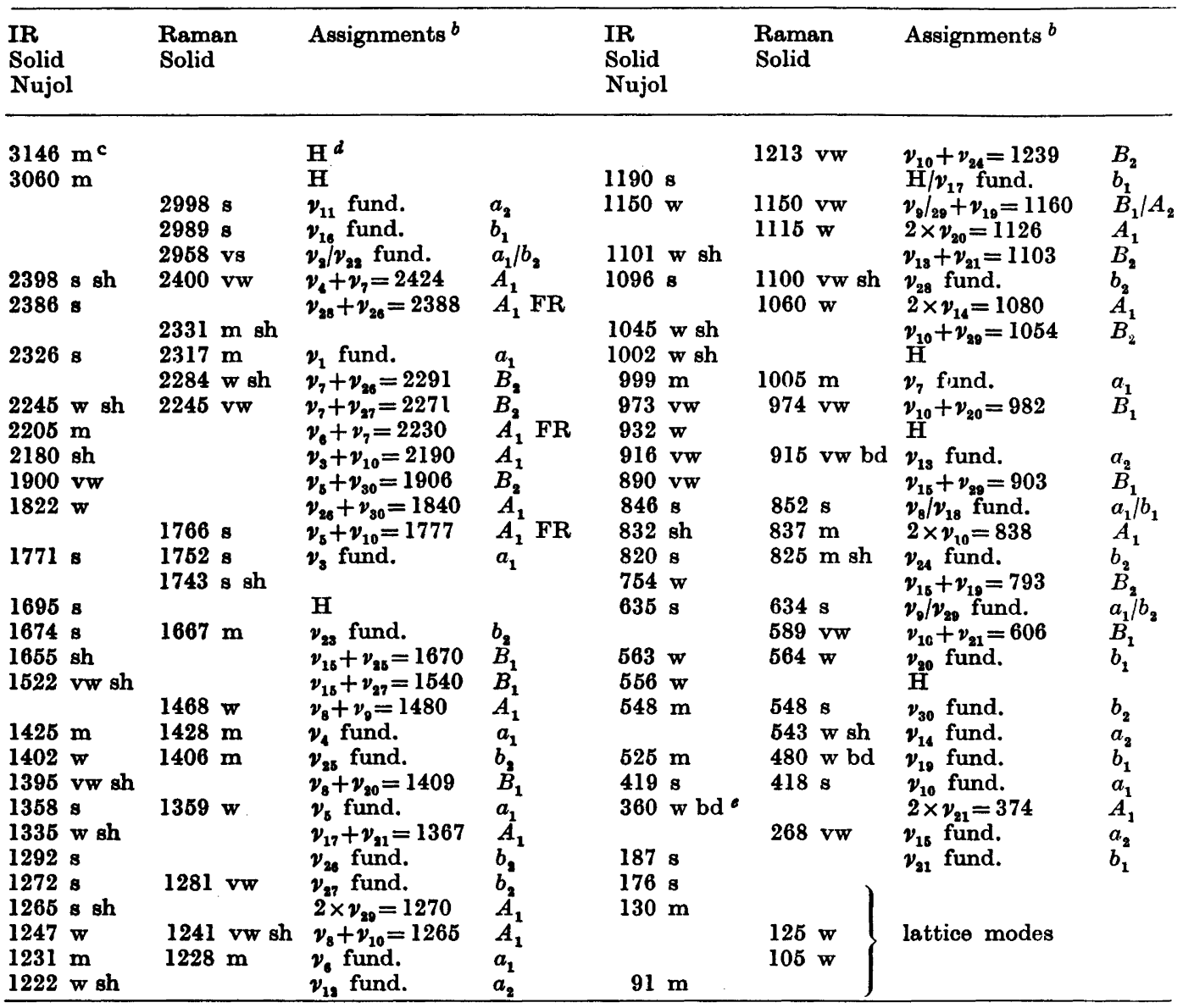

a The weak infrared and Raman bands in the regions $5000-3200$ and $2800-2500 \mathrm{~cm}^{-1}$ are omitted. ${ }^{b}$ When available the combination frequencies and overtones are calculated from solid state data. ${ }^{c}$ For abbreviations used, see footnotes to Table $1 .{ }^{d} \mathrm{H}=$ bands assigned to succinimide. ' Solid state frequencies below $400 \mathrm{cma}^{1}$ are from polyethylerie (Rigidex) pellet.

to bands around $1400 \mathrm{~cm}^{-1}$ in accordance with the observed dichroism. Correspondingly, the wagging fundamentals $v_{6}$ and $v_{26}$ were observed at 1238 and $1294 \mathrm{~cm}^{-1}$ (SIM) and 1231 and $1292 \mathrm{~cm}^{-1}$ (SIMD).

The $1373 \mathrm{~cm}^{-1}$ band which was clearly of species $a_{1}$ involves predominantly CNC stretch, close to the corresponding frequencies for the maleimides., ${ }^{2,3}$ An infrared band at 1416 (SIM) and $820 \mathrm{~cm}^{-1}$ (SIMD) was interpreted as the $\mathbf{N}-\mathbf{H}(\mathbf{D})$ in-plane bend. In agreement with the observed dichroism and Raman polarization data, the $1225 \mathrm{~cm}^{-1}$ band (SIM) is taken as the
$\mathrm{CH}_{2}$ twist of species $a_{2}\left(v_{12}\right)$. The corresponding $b_{1}$ mode $\left(v_{17}\right)$ is assigned to the band at 1180 $\mathrm{cm}^{-1}$ partly overlapping the $b_{2}$ fundamental $\left(v_{27}\right)$, which involves the CNC stretch. Similar bands are found for SIMD at 1222, 1190 and $1272 \mathrm{~cm}^{-1}$, respectively.

Three skeletal modes $\left(v_{7}, v_{28}, v_{8}\right)$ with strong contributions from skeletal $\mathrm{C}-\mathrm{C}$ stretch are found at 1001, 935 and $850 \mathrm{~cm}^{-1}$ for SIM and at 999,1096 and $846 \mathrm{~cm}^{-1}$ for SIMD. For SIMD as well as for $N$-deuteriomaleimide ${ }^{2}$ the $b_{2}$ modes of the region $1300-800 \mathrm{~cm}^{-1}$, not localized to the methylene groups, are highly

Acta Chem. Scand. A 30 (1976) No. 7 
Table 3. Observed $\left(\nu_{0}\right)$ and calculated $\left(\nu_{c}\right)$ fundamentals for succinimide and $N$-deuteriosuccinimide.

\begin{tabular}{|c|c|c|c|c|c|}
\hline \multirow{2}{*}{\multicolumn{2}{|c|}{$\begin{array}{l}\text { Species } \\
\text { and No. }\end{array}$}} & \multicolumn{2}{|c|}{ Succinimide } & \multicolumn{2}{|c|}{$N$-Deuteriosuccinimide } \\
\hline & & $\overline{v_{0} a}$ & $\overline{\nu_{c}}$ & $\overline{v_{0} a}$ & $\nu_{\mathrm{c}}$ \\
\hline \multirow[t]{10}{*}{$a_{1}$} & $\nu_{1}$ & 3150 & 3151 & 2326 & 2331 \\
\hline & $v_{2}$ & 2960 & 2946 & 2958 & 2946 \\
\hline & $v_{3}$ & 1772 & 1773 & 1771 & 1772 \\
\hline & $v_{4}$ & 1428 & 1412 & 1425 & 1412 \\
\hline & $\nu_{5}$ & 1373 & 1403 & 1358 & 1384 \\
\hline & $\nu_{8}$ & 1238 & 1239 & 1231 & 1231 \\
\hline & $\nu_{7}$ & 1001 & 973 & 999 & 972 \\
\hline & $v_{8}$ & 850 & 839 & 846 & 831 \\
\hline & $\nu_{0}$ & 640 & 640 & 635 & 637 \\
\hline & $v_{10}$ & 427 & 414 & 419 & 413 \\
\hline \multirow{5}{*}{$a_{2}$} & $v_{11}$ & 3000 & 2998 & 2998 & 2998 \\
\hline & $v_{12}^{11}$ & 1224 & 1190 & 1222 & 1190 \\
\hline & $\nu_{13}$ & 935 & 1064 & 916 & 1064 \\
\hline & $\nu_{14}$ & 537 & 538 & 543 & 538 \\
\hline & $v_{15}$ & 267 & 292 & 268 & 292 \\
\hline \multirow[t]{6}{*}{$b_{1}$} & $v_{16}$ & 2990 & 2988 & 2989 & 2988 \\
\hline & $v_{17}$ & 1180 & 1197 & 1190 & 1197 \\
\hline & $v_{18}$ & 844 & 749 & 846 & 768 \\
\hline & $\nu_{10}$ & 823 & 860 & 525 & 636 \\
\hline & $v_{20}$ & 563 & 536 & 563 & 534 \\
\hline & $\nu_{21}$ & 190 & 184 & 187 & 177 \\
\hline \multirow[t]{9}{*}{$b_{2}$} & $v_{22}$ & 2946 & 2946 & - & 2946 \\
\hline & $\nu_{23}$ & 1697 & 1698 & 1674 & 1687 \\
\hline & $\nu_{24}$ & 1416 & 1427 & 820 & 814 \\
\hline & $\nu_{25}$ & 1402 & 1403 & 1402 & 1403 \\
\hline & $\nu_{26}$ & 1294 & 1310 & 1292 & 1334 \\
\hline & $\nu_{27}$ & 1192 & 1173 & 1272 & 1234 \\
\hline & $\nu_{28}$ & 935 & 941 & 1096 & 1140 \\
\hline & $\nu_{28}$ & 650 & 666 & 635 & 656 \\
\hline & $\nu_{30}$ & 556 & 553 & 548 & 545 \\
\hline
\end{tabular}

a When possible, frequencies are taken from the infrared spectra of the solids.

mixed compared to the parent molecules, and therefore causing erratic isotopic shifts.

The $\mathrm{CH}_{2}$ rock of species $a_{2}\left(v_{13}\right)$ was found between 935 and $908 \mathrm{~cm}^{-1}$ for SIMD and the $N$-halogenated succinimides." For SIM this fundamental presumably overlaps the more intense $b_{2}$ mode at $935 \mathrm{~cm}^{-1}$.

For SIM three overlapping bands are situated between 850 and $820 \mathrm{~cm}^{-1}$. From comparison with the $N$-halogenated succinimides ${ }^{4}$ the bands at $844 \mathrm{~cm}^{-1}$ are considered as the $\mathrm{CH}_{2}$ rock $\left(v_{18}\right)$ of species $b_{1}$, while the stronger lines at $823 \mathrm{~cm}^{-1}$ are attributed to the $\mathrm{N}-\mathrm{H}$ out-ofplane bend $\left(v_{19}\right)$. For SIMD the $\mathrm{CH}_{2}$ rock of species $b_{1}$ overlaps the $a_{1}$ fundamental $v_{8}$ at $846 \mathrm{~cm}^{-1}$. The $\mathrm{N}-\mathrm{D}$ bending mode of species $b_{1}$, is situated at $525 \mathrm{~cm}^{-1}$ and corresponds to the band at $518 \mathrm{~cm}^{-1}$ for $N$-deuteriomaleimide. ${ }^{2}$

In complete agreement with the results for the maleimides 2,3 the succinimides have two bands in the region $640-680 \mathrm{~cm}^{-1}$ taken as $v_{9}\left(a_{1}\right)$ and $v_{29}\left(b_{2}\right)$, the former being more intense in the Raman spectrum.

Weak bands at 537 and $543 \mathrm{~cm}^{-1}$ for SIM and SIMD, respectively, are attributed to a skeletal mode $\left(\nu_{14}\right)$ of species $a_{2}$ while the corresponding $b_{1}$ fundamental $\left(v_{20}\right)$ is assigned to the band $563 \mathrm{~cm}^{-1}$ for both molecules.

For SIM the $563 \mathrm{~cm}^{-1}$ band presumably overlaps a fundamental $\left(v_{30}\right)$ of species $b_{2}$, mainly involving the asymmetrical bending of the $\mathrm{C}=\mathrm{O}$ groups. The corresponding $a_{1}$ mode $\left(v_{10}\right)$ is assigned to the strong infrared and Raman bands at $427 \mathrm{~cm}^{-1}$ in good agreement with the results from the maleimides. ${ }^{2}$ Similar bands are found for SIMD at 548 and $419 \mathrm{~cm}^{-1}$, respectively.

The remaining two fundamentals $v_{15}\left(a_{2}\right)$ and $v_{21}\left(b_{1}\right)$ are attributed to the bands at 265 and $190 \mathrm{~cm}^{-1}$ for SIM and at 268 and $187 \mathrm{~cm}^{-1}$ for SIMD, in good agreement with the results for the maleimides..$^{2,3}$

\section{NORMAL COORDINATE ANALYSIS}

Detailed knowledge about the harmonic force constants cannot be obtained for SIM and the $N$-halogenated succinimides ${ }^{4}$ independently since the information from the normal vibrations is insufficient for this purpose. Also, the ambiguities in the assignments suggested us to use force constant calculations as a tool for obtaining more reliable assignments rather than determining force constants of general physical significance. Therefore, the scope of this section is to demonstrate that the approximation of transferable force constants may be applied in establishing reliable spectral interpretations of SIM, SIMD as well as the $N$-halogenated succinimides."

The normal coordinate analysis was carried out employing an approximate internal valence force field (IVFF), and the least squares method was applied in adjusting the force constants. As a starting point we used the force fields for maleimide ${ }^{2}$ and succinic anhydride. ${ }^{10}$ The related structure and spectral similarities of all the succinimides $(\mathrm{N}-\mathrm{H}, \mathrm{N}-\mathrm{D}, \mathrm{N}-\mathrm{Cl}$,

Acta Chem. Scand. A 30 (1976) No. 7 
Table 4. Final valence force constants for succinimide.

\begin{tabular}{|c|c|c|}
\hline \multirow[t]{2}{*}{ Force type } & \multicolumn{2}{|l|}{ Constants } \\
\hline & $\overline{\text { Symbol }^{a}}$ & Value $^{b}$ \\
\hline stretch & $\begin{array}{l}K_{\mathrm{a}} \\
\boldsymbol{K}_{\mathrm{s}} \\
\boldsymbol{K}_{\mathrm{r}} \\
\boldsymbol{K}_{\mathrm{d}} \\
\boldsymbol{K}_{1} \\
\boldsymbol{K}_{\mathrm{b}}\end{array}$ & $\begin{array}{c}5.297 \\
11.611 \\
3.934 \\
3.5 \\
4.798 \\
5.456\end{array}$ \\
\hline stretch-stretch & 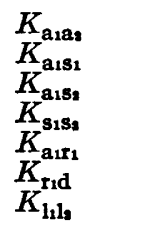 & $\begin{array}{r}1.045 \\
1.53 \\
-0.04 \\
0.3 \\
1.087 \\
0.211 \\
-0.079\end{array}$ \\
\hline bend & $\begin{array}{l}H_{\eta} \\
H_{\xi} \\
H_{\psi} \\
H_{\phi} \\
H_{\theta} \\
H_{\alpha} \\
H_{8} \\
H_{\beta} \\
H_{\mu}\end{array}$ & $\begin{array}{l}1.185 \\
1.185 \\
0.574 \\
0.574 \\
0.538 \\
1.344 \\
1.079 \\
1.566 \\
0.47\end{array}$ \\
\hline stretch-bend & $\begin{array}{l}F_{\mathrm{a} \eta} \\
F_{\mathrm{r} \xi} \\
F_{\mathrm{s} \alpha} \\
F_{\mathrm{r} \psi} \\
\boldsymbol{F}_{\mathrm{d} \phi}\end{array}$ & $\begin{array}{r}0.17 \\
0.17 \\
-1.19 \\
0.15 \\
0.15\end{array}$ \\
\hline bend-bend & $\begin{array}{l}F_{\boldsymbol{w}_{1} \boldsymbol{\psi}_{2}} \\
\boldsymbol{F}_{\phi_{1} \phi_{2}} \\
F_{\psi_{1} \phi_{1}}\end{array}$ & $\begin{array}{l}-0.038 \\
-0.038 \\
-0.015\end{array}$ \\
\hline outo $^{c}$ & $\begin{array}{l}O_{\pi_{1}} \\
O_{\pi_{3}}\end{array}$ & $\begin{array}{l}0.411 \\
0.363\end{array}$ \\
\hline outo-outo & $O_{\pi_{1} \pi_{2}}$ & 0.086 \\
\hline torsion & $T_{\tau}$ & 0.931 \\
\hline outo-torsion & $\begin{array}{l}F_{\pi_{1} \tau_{1}} \\
F_{\pi_{2} \tau_{1}}\end{array}$ & $\begin{array}{r}-0.118 \\
0.173\end{array}$ \\
\hline torsion-torsion & $T_{\tau_{1} \tau_{3}}$ & -0.031 \\
\hline
\end{tabular}

${ }^{a}$ For meaning of symbols, see Fig. 2. ${ }^{b}$ In units of mdyn $\AA^{-1}$ (stretch constants), mdyn rad $^{-1}$ (stretchbend interaction) and mdyn $\AA \operatorname{rad}^{-2}$ (bending and torsion constants). $c$ outo means out-of-plane bending.

$\mathrm{N}-\mathrm{Br}, \mathrm{N}-\mathrm{I}$ ) justified the use of transferable force constants. We therefore employed data from all the five compounds simultaneously in the least squares fitting, keeping the force constants concerning the $\mathrm{H}_{2}-\mathrm{C}-\mathrm{C}-\mathrm{H}_{2}$ part of the molecule at the same value for all.
Moreover, we decided to give the $\mathrm{C}=\mathrm{O}$ bending force constants and some of the interaction constants a common value for all the compounds. To reduce the number of parameters further we have made $H_{\eta}$ and $H_{\xi}, H_{\varphi}$ and $H_{\phi}$, $F_{\text {a } \eta}$ and $F_{\mathrm{r} \xi}, F_{\mathrm{r} \varphi}$ and $F_{\mathrm{d} \phi}, F_{\psi_{1} \varphi_{2}}$ and $F_{\phi_{1} \phi_{2}}$ equal. The final force field consisted of 81 parameters. It was reported ${ }^{11}$ that for similar molecules the $\mathrm{C}=\mathrm{O}$ stretching frequencies can be calculated correctly by using interaction terms between the two $\mathrm{C}=\mathrm{O}$ bonds, the two $\mathrm{C}-\mathrm{N}$ bonds and between the $\mathrm{C}=\mathrm{O}$ and $\mathrm{C}-\mathrm{N}$ bonds. This model force field was also suitable for the succinimides. For the remaining interaction constants we have only introduced terms between neighbouring groups.

Since the most complete data were obtained for the solid state, we have employed these frequencies in the calculations leading to even more approximate force constants due to crystal effects. The agreement between observed

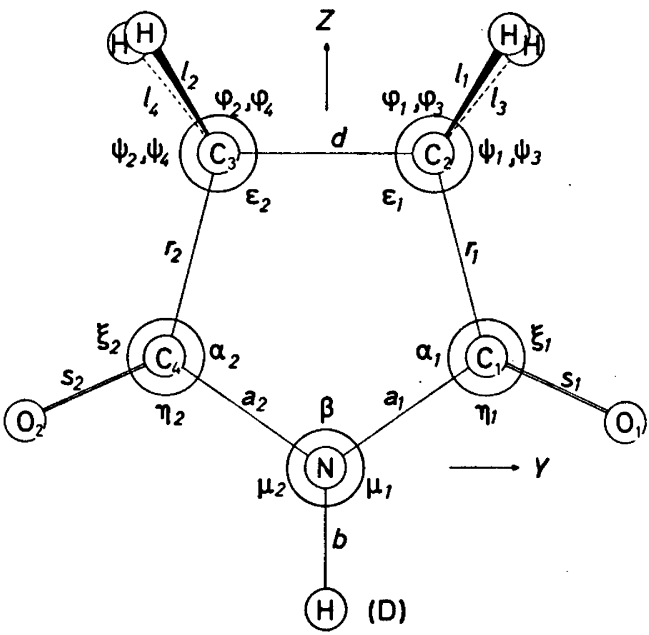

Fig. 2. Internal coordinates for succinimide. The following coordinates are not shown in the figure: out-of-plane bending: $\pi_{1}=$ $\pi\left(\mathrm{NC}_{2} \mathrm{C}_{1} \mathrm{O}_{1}\right), \quad \pi_{2}=\pi\left(\mathrm{C}_{3} \mathrm{NC}_{4} \mathrm{O}_{2}\right), \quad \pi_{3}=\pi\left(\mathrm{C}_{4} \mathrm{C}_{1} \mathrm{NH}\right)$; torsion: $\tau_{1}=\tau\left(\mathrm{NC}_{1} \mathrm{C}_{2} \mathrm{C}_{3}\right), \quad \tau_{2}=\tau\left(\mathrm{NC}_{4} \mathrm{C}_{3} \mathrm{C}_{2}\right)$. The out-of-plane bending and torsion coordinates are defined as in Wilson, Decius and Cross, ${ }^{12}$ where the succession of the letters corresponds to atom $2,3,4,1$ in the definition of the outof-plane bending coordinate. $\theta_{1}, \theta_{2}$ : the bending of the angles $\mathrm{HCH}$. The following structural parameters were assumed: $\mathrm{CN}$ : $1.379 ; \mathrm{C}_{1}-\mathrm{C}_{2}$ : 1.477; $\mathrm{C}_{2}-\mathrm{C}_{3}: 1.520 ; \mathrm{C}=\mathrm{O}: 1.220 ; \mathrm{C}-\mathrm{H}: 1.09$; $\mathrm{N}-\mathrm{H}: 0.99 \AA ; \angle C N C: 110.0^{\circ} ; \angle \mathrm{NCC}: 110.5^{\circ}$; $\angle \mathrm{NCO}: 122.9^{\circ}$ and $\angle \mathrm{C}_{1} \mathrm{C}_{2} \mathrm{H}$ : $116.1^{\circ}$.

Acta Chem. Scand. A 30 (1976) No. 7 
and calculated frequencies was quite good for the $a_{1}$ and $b_{2}$ species, while some large discrepancies still remained for the out-of-plane modes. This may very well be due to large amplitudes of certain out-of-plane vibrations as mentioned in Ref. 9. For the $a_{2}$ and $b_{1}$ fundamentals (except those involving the $\mathrm{CH}_{2}$ groups) we have employed an almost complete force field, using the approximation of keeping some of the interaction constants common for all the molecules. Since the assign. ments for these species are not as certain as for the in-plane modes and the force constants not as physically interesting, we do not con. sider it worth while to make any further calculations to achieve a better fit. Later, we will expand the calculations with more data from succinic anhydride and the $C$ deuterated species of both succinimide and succinic anhydride.

The internal in-plane coordinates are shown in Fig. 2, and the out-of-plane coordinates are given in the underlying text. Our calculated fundamentals are listed together with the observed frequencies in Table 3 . The final set of force constants for SIM and SIMD are given in Table 4. The results seem physically reason. able, although the restrictions imposed, keeping some of the force constants transferable to the halogenated molecules and using as few constants as possible, are reflected in some of the values. The potential energy distribution (PED) of the fundamentals among the valence coordinates were calculated. These data were not included in Table 3 for the sake of brevity, but can be obtained from the authors on request.

Acknowledgement. The authors are grateful to K. Ruzicka for the preparative work. Financial support was received from the Norwegian Research Council for Science and the Humanities.

\section{REFERENCES}

1. Rogstad, A., Klæboe, P., Baranska, H., Bjarnov, E., Christensen, D. H., Nicolaisen, F., Nielsen, O. F., Cyvin, B. N. and Cyvin, S. J. J. Mol. Struct. 20 (1974) 403.

2. Woldbæk, T., Klæboe, P. and Nielsen, C. J. J. Mol. Struct. 27 (1975) 283.

3. Woldbæk, T., Klæboe, P. and Nielsen, C. J. J. Mol. Struct. 28 (1975) 269.

Acta Chem. Scand. A 30 (1976) No. 7
4. Woldbæk, T., Klæboe, P. and Christensen, D. H. Acta Chem. Scand. A 30 (1976) 547.

5. Uno, T. and Machida, K. Bull. Chem. Soc. Jpn. 35 (1962) 276.

6. Hayashii, S. Bull. Inst. Chem. Res. Kyoto Univ. 43 (1965) 355.

7. Mason, R. Acta Crystallogr. 14 (1961) 720; 9 (1956) 405.

8. Brown, R. N. Acta Crystallogr. 14 (1961) 711.

9. Brendhaugen, K., Kolderup Fikke, M. and Seip, H. M. Acta Chem. Scand. 27 (1973) 1101.

10. Di Lauro, C., Califano, S. and Adembri, G. J. Mol. Struct. 2 (1968) 173.

11. Colthup, N. B. and Orloff, M. K. Spectrochim. Acta Part A 30 (1974) 425.

12. Wilson, E. B., Decius, J. C. and Cross, C. Molecular Vibrations, McGraw-Hill, New York, Toronto, London 1955, p. 56.

Received January 16, 1976. 